\title{
Kräfte zwischen Stoff und Geist - Über die zerronnenen Träume der Homöopathie
}

\section{Michael Rüegga}

a Der Verfasser studierte von 1999 bis 2006 an der Universität Zürich Philosophie, Religionswissenschaft und Osteuropäische Geschichte. Beim Artikel handelt es sich um eine Zusammenfassung seiner gleichnamigen Lizentiatsarbeit, die auf eigenen Erfahrungen mit dem Gegenstand beruht: Bis April 2005 arbeitete der Verfasser neben dem Studium als Naturheilpraktiker mit kantonaler Bewilligung in St. Gallen.
Korrespondenz:

lic. phil. Michael Rüegg Kronbergstrasse 10 CH-9000 St. Gallen michaelrueegg@gmx.ch

\section{Einleitung}

Die Homöopathie erhebt Anspruch auf strenge Wissenschaftlichkeit. In eigentümlichem Kontrast dazu stehen ihre zwei grossen metaphysischen Prinzipien: das Ähnlichkeitsgesetz (Similia similibus curentur) und die Arzneikraftentwicklung (Potenzierung). Diese Ausführungen werden sich auf eine Betrachtung des zweiten Prinzips beschränken; zum einem deshalb, weil in der Streitfrage um die wissenschaftliche Legitimation der Homöopathie das - wohl nicht weniger fragwürdige - Ähnlichkeitsgesetz eine völlig untergeordnete Rolle spielt, zum anderen, weil die hier dargestellte Problematik im Zusammenhang mit unfassbaren Kräften in vielerlei Hinsicht exemplarisch ist und auch andere ganzheitliche Konzepte berührt, z.B. die anthroposophische oder die chinesische Medizin.

Das Problem der Arzneikraftentwicklung Das bis heute im Kern unverändert gültige Verfahren zur Herstellung homöopathischer Arzneimittel verdankt sich dem Begründer der Homöopathie, Samuel Hahnemann (1755-1843). Durch ein kombiniertes mechanisches Prozedere der schrittweisen Verdünnung und Verreibung bzw. Verschüttelung können «geistartige Arzneikräfte» aus jeder beliebigen «rohen Substanz» entwickelt werden. Entscheidend ist dabei aber weniger die Verdünnung als vielmehr der sie begleitende Vorgang der Verreibung bzw. Verschüttelung das eigentliche Potenzieren. Hahnemann gibt uns davon in seinem Hauptwerk «Organon der Heilkunst» im § 269 folgende Erläuterung: «Die homöopathische Heilkunst entwickelt zu ihrem besondern Behufe, die innern, geistartigen Arzneikräfte der rohen Substanzen mittels einer ihr eigenthümlichen, bis zu meiner Zeit unversuchten Behandlung, zu einem, früher unerhörten Grade, wodurch sie sämmtlich erst recht sehr, ja unermesslich-durchdringend wirksam und hülfreich werden, selbst diejenigen unter ihnen, welche im rohen Zustande nicht die geringste Arzneikraft im menschlichen Körper äussern. Diese merkwürdige Veränderung in den Eigen-

\section{Homéopathie:}

\section{rêves évanouis d'une force}

\section{entre matière et esprit}

L'article que nous publions montre qu'il est extrêmement productif $d$ 'introduire un élément philosophique dans la question relative à l'aspect scientifique de l'homéopathie. Jusqu'à présent, le débat sur le sens et l'utilité de l'homéopathie s'est déroulé principalement dans le cadre des sciences empiriques. Les critiques portent avant tout sur le procédé mécanique de fabrication des médicaments homéopathiques, c'est-à-dire sur la manière avec laquelle la force médicamenteuse se développe (dynamisation) en contredisant visiblement les lois naturelles connues. Les partisans de I'homéopathie répondent à ce manque de cohérence par des arguments avant-gardistes et font référence à la physique quantique, à la théorie du chaos ou à la cybernétique. Dans ce litige marqué par l'empirisme, la problématique devient encore plus complexe quand la philosophie s'empare des présupposés théoriques des forces médicamenteuses homéopathiques. Outre la question des contradictions possibles, il suffit de comparer la définition de la force en physique et en homéopathie pour voir l'urgence d'une information dans ce champ de tensions entre savoir et pseudoscience.

schaften der Naturkörper, durch mechanische Einwirkung auf ihre kleinsten Theile, durch Reiben und Schütteln entwickelt die latenten, vorher unmerklich, wie schlafend in ihnen verborgen gewesenen, dynamischen Kräfte [...]. Man nennt daher diese Bearbeitung derselben 
Dynamisiren, Potenziren (Arzneikraft-Entwickelung)» [1].

Diese mechanische Arzneikraftentwicklung widerspricht offenkundig den bekannten Naturgesetzen. Denn Prozesse wie Verreibung und Verschüttelung können keine Änderungen physikalisch-chemischer Grössen bewirken [2]. Von einem wissenschaftlichen Standpunkt aus betrachtet handelt es sich bei homöopathischen Arzneien um nichts anderes als Verdünnungen. Damit ergibt sich ein Problem: Ab einem gewissen Grad der Verdünnung - die Grenze wird bestimmt durch die Avogadro-Konstante - enthält eine homöopathische Arznei keine Moleküle der Ausgangssubstanz mehr (sogenannte Hochpotenzen ab der D24 bzw. der C12). Eine gemäss naturwissenschaftlichem Ermessen nichtwirksame Arznei ist ein sprichwörtlicher Etikettenschwindel: Was auf einem Fläschchen draufsteht (z. B. Natrium chloratum C30), lässt sich im Fläschchen nicht nachweisen. Wer immer Hochpotenzen anwendet, gerät somit in eine, wie es der Physiker Lambeck formuliert, «erklärungsfordernde Spannung» [2].

Die Befürworter der Homöopathie antworten auf diese Spannung mit einer Vielzahl schwer fassbarer Argumente. Prototypisch sind die Verweise auf Quantenphysik, Chaostheorie und Kybernetik. Der wohl populärste zeitgenössische Homöopath und Träger des Wright Livelihood Award (besser bekannt unter dem Namen alternativer Nobelpreis), Georgos Vithoulkas [3], stützt sich genauso wie auch Vertreter der Kollegialen Instanz für Komplementärmedizin der Universität Bern (KIKOM) [4, 5] auf die moderne Physik bzw. neuere Informationstheorien, um die Erklärungslücken der Homöopathie aufzufüllen. Die Folgen dieser avantgardistischen Rechtfertigungsstrategien sind bekannt: die Homöopathiedebatte endet - aus Mangel an experimentell überprüfbaren Theorien - in ermüdenden statistischen Scheingefechten.

Im Folgenden soll gezeigt werden, dass ein philosophischer Zugang, der nicht das uferlose Material der empirischen Forschung in den Mittelpunkt stellt, sondern die theoretischen Voraussetzungen, mehr Aussicht auf Orientierung innerhalb der festgefahrenen Debatte über Sinn und Nutzen der Homöopathie verspricht.

b Andere Lösungsansätze (etwa in Richtung eines Aspektdualismus) kommen hier nicht in Frage, weil in der Lehre von der Arzneikraftentwicklung ja gerade eine mechanische Trennung zweier ungleicher Substanzen propagiert wird.
Auf dem Prüfstand steht die Behauptung, dass es 1. möglich ist, durch ein mechanisches Verfahren eine geistartige Arzneikraft aus einer stoffen Ausgangssubstanz zu gewinnen und dann auf einen stofflichen Träger (Milchzucker oder Alkohol) zu binden; 2. die so hergestellte homöopathische Arznei auf den üblichen Applikationswegen (primär die Mundschleimhaut) ihre Wirkung entfaltet.

Fokussiert man auf die begrifflichen Voraussetzungen, ergeben sich zwei Anfragen: Die eine betrifft den Gebrauch des Wortes geistartig, die andere den Gebrauch des Kraftbegriffs.

Die erste Anfrage, die Rede von etwas Geistartigem, berührt ein altes Problem: den Zusammenhang von Stoff und Geist bzw. von Körper und Seele. Oder um mit Kant zu sprechen: Hier verbirgt sich «ein verwickelter metaphysischer Knoten, den man nach Belieben auflösen oder abhauen kann» [6]. Mit anderen Worten gibt es genau zwei Ansätze, um die Hahnemannsche Arzneikraftentwicklung zu deuten: einen dualistischen und einen reduktionistischen. ${ }^{\mathrm{b}}$

Im ersten Fall ist zu fragen, was etwas Geistartiges von etwas Stofflichem überhaupt unterscheidet. Man kann sich beispielsweise eine Weinflasche denken, die mit etwas Geistigem aufgefüllt wird. Würde sich nun das Gewicht der Flasche ändern? Oder wäre die Flasche irgendwann voll? Wohl nein. Denn in diesem Fall würden wir das in die Flasche Hineingefüllte ohne jeden Zweifel etwas Stoffliches nennen, z.B. Wein. Folglich muss man annehmen, dass man etwas Geistiges in diese Flasche füllen könnte, ohne jede Befürchtung, dadurch kostbaren Wein zu verdrängen, und auch ohne jede Sorge, dadurch dem Flaschengeist die Füsse nass zu machen. Das Fazit ist knapp und bündig: Entweder sind etwas Stoffliches und etwas Geistartiges zwei ganz und gar ungleiche Dinge (das eine wäre nämlich ausgedehnt und den physikalischen Gesetzen unterworfen, das andere dagegen ausdehnungslos und nicht den physikalischen Gesetzen unterworfen), oder diese Unterscheidung in Stoffliches und Geistartiges ist ohne jeden Sinn [6].

Unter dieser dualistischen Voraussetzung aber löst sich die Arzneikraftentwicklung in einem Widerspruch auf. Es ist denkunmöglich, durch ein mechanisches Verfahren etwas Geistartiges (das nicht den physikalischen Gesetzen unterworfen ist) aus etwas Stofflichem (das den physikalischen Gesetzen unterworfen ist) herauszulösen und an einen stofflichen Träger zu binden. Mit anderen Worten: Eine mechanische Bearbeitung kann immer nur physikalische, nie aber geistartige Veränderungen hervorbringen. Dieser Widerspruch bestätigt sich, sobald man auf die Folgen achtgibt. Hätte das homöopathische Verfahren unter dieser Voraussetzung den Status eines Naturgesetzes, dann wäre mittels der Arzneikraftentwicklung ein Kunststück möglich geworden, das bislang noch keinem Chirurgen 
gelungen ist: den menschlichen Geist vom stofflichen Körper $\mathrm{zu}$ trennen und sozusagen auf Erden bzw. auf Milchzucker unsterblich zu machen.

Aus diesem Widerspruch kann man sich nur retten, wenn man den metaphysischen Knoten abhaut. Das ist im zweiten Ansatz der Fall. Man reduziert das Geistartige auf etwas Stoffliches, so dass es auch den physikalischen Gesetzen unterworfen ist. Das Resultat dieser Kastration ist folglich ein handfester Reduktionismus, in dem alle Unterschiede zwischen Stoff und Geist aufgehoben sind [6]. Wie gesagt, ist in diesem Fall aber auch jede Rede von geistigartigen Kräften, die man aus einer rohen, d.h. stofflichen Ausgangssubstanz gewinnen kann, sinnlos geworden.

Die zweite Anfrage, die Rede von (geistartigen) Arzneikräften, die bei der Herstellung von Stoff zu Stoff bzw. bei der Anwendung von Stoff zu Mensch übertragbar sind, berührt ein ontologisches Problem: das Verhältnis dieser Kräfte zur Wirklichkeit. Im Vergleich mit der Physik und ihrem Kraftbegriff wird erkennbar, dass die Annahme geistartiger Kräfte auf einem vorwissenschaftlichen Wirklichkeitsverständnis beruht.

Die Naturwissenschaften zeigen einen Wandel von einem stofflich-substantiellen zu einem funktionell-symbolischen Wirklichkeitsverständnis [7]. So ist Newtons Kraftbegriff noch eine ontologisch eigenständige Grösse in der Natur, die ein subjekthaftes Wesen nahe legt [8]. Dieses stoffliche Verständnis der klassischen Mechanik wird sichtbar in der Annahme von Imponderabilien. Man spricht bis ins beginnende 19. Jahrhundert hinein von einem Licht- und einem Wärmestoff, von elektrischer und magnetischer Materie [7]. Die Äthertheorie des Lichts wird sogar erst durch Einsteins Arbeiten obsolet.

Die Ablösung dieses mechanischen Weltbildes beginnt in der zweiten Hälfte des 19. Jahrhunderts [9]. In einem fortlaufenden Prozess der Mathematisierung gewinnt die Physik der Moderne schliesslich ein neues Wirklichkeitsverständnis. ${ }^{c}$ Physikalische Theorien sind nicht mehr Abbilder der Natur, sondern funktionellsymbolisch formulierte Ordnungen, die sich den Ordnungen der Erscheinungen annähern, also immer falsifizierbar bleiben. Und so tritt etwa der Kraftbegriff als eine methodologisch zwar nach wie vor sinnvolle, aber nicht notwendige Grösse in der modernen Physik auf [8].

Offenkundig besteht zwischen diesen grossen begrifflichen Anstrengungen der Physik und der bis heute im Kern unveränderten Lehre von der Arzneikraftentwicklung eine grundlegende
Differenz. Letztere ist bei einem stofflichen Wirklichkeitsverständnis stehen geblieben. Denn die mechanisch erzeugbaren Kräfte der Homöopathie sind nicht nur - analog Newtons Kraftbegriff ontologisch eigenständige Grössen, die von Ding zu Ding übertragbar sind (Ausgangssubstanz/ Trägerstoff und Arznei/Mensch), sondern darüber hinaus je nach Ausgangssubstanz mit spezifischen Eigenschaften ausgestattet. Kulturphilosophisch gesehen entspricht diese Vorstellung von individuellen Kräften einer magischen Entwicklungsstufe [7]. ${ }^{\mathrm{d}}$

Diese begrifflichen Schwierigkeiten - sowohl im Gebrauch des Wortes geistartig als auch im Gebrauch des Kraftbegriffs - sind Ausgangspunkt einer Pathogenese, die bis in die Gegenwart andauert. Denn alle modernen Erklärungsversuche der homöopathischen Arzneikräfte werden letztlich auf diesen widersprüchlichen Grund zurückgeworfen.

\section{Moderne Erklärungsversuche}

Am Übergang vom 19. zum 20. Jahrhundert kann man erstmals naturwissenschaftlich orientierte Erklärungsversuche beobachten. Die homöopathischen Kräfte werden nicht mehr mit vitalistischen (Hahnemann), sondern mit neuentdeckten physikalisch-energetischen Grössen identifiziert. So spricht etwa der nach Hahnemann wohl berühmteste - und als Anhänger der Lehre Swedenborgs auch umstrittenste - homöopathische Arzt, der US-Amerikaner James Tyler Kent (1849-1916), von «strahlender Form der Materie» [11]. In seinem Rückgriff auf die sich mächtig entfaltende Physik wird ein - notabene ungültiger - Argumentationstyp sichtbar, der bis in die Gegenwart alle Rechtfertigungsversuche der Befürworter ganzheitlicher Konzepte beherrscht: die Gleichsetzung physikalischer und nichtphysikalischer Begriffe. ${ }^{e}$

Was ist aber mit dieser Flucht in eine physikalisch-energetische Lesart der unfassbaren Kräfte gewonnen? Im Licht der dargestellten begrifflichen Schwierigkeiten ergeben sich zwei Schlussfolgerungen:

Erstens endet jeder Versuch, die homöopathischen Arzneikräfte mittels avantgardistischer Argumente aus dem Bereich moderner Theorien (Quantenphysik, Chaostheorie, Kybernetik etc.) zu legitimieren, in einem - erkenntnistheoretisch ebenso naiven wie unhaltbaren - materialistischen Reduktionismus. Damit aber gerät die Homöopathie in einen performativen Widerspruch: denn in ihrem ganzheitlichen Selbstverständnis, das gerade eine Überwindung der Begriffen identifiziert (Geist und Materie). 
materialistisch-wissenschaftlichen Medizin verspricht, führt sie sich mit diesem Akt der Geistlosigkeit eigenhändig ad absurdum.

Zweitens erwächst aus der Diskrepanz zwischen wissenschaftlichem Anspruch und dinglichem Wirklichkeitsverständnis ein eigentümliches Zwitterwesen [7], das sich terminologisch gesehen zwar immer nach der neuesten wissenschaftlichen Mode kleidet, d. h. heute im Gewand von Energien und morgen von kybernetischen Informationen usw. erscheint, aber von seiner Natur her doch ganz einem magischen Grund verhaftet bleibt. Mit diesem Spagat zwischen Wissen und Scheinwissen gerät die Homöopathie nicht nur in Konflikt mit den Naturwissenschaften, sondern immer wieder in die Nähe esoterisch-gnostischer Bewegungen (z. B. Swedenborgkirche, Christian Science, Anthroposophie) und damit auch in Konflikt mit unserem aufgeklärten, jüdisch-christlich geprägten Weltbild.

\section{Erbaulicher Ausblick}

Zieht man Bilanz, ist die Diagnose ernüchternd. Die Arzneikraftentwicklung offenbart sich als ein ebenso unwissenschaftliches wie widersprüchliches Verfahren. Will man nicht hinter wesentliche Errungenschaften der Aufklärung zurückfallen, kann man nur anraten, künftig auf alles mechanische Beiwerk zu verzichten. Diesen Verzicht kann man auf zwei Arten denken: einmal wissenschaftlich und einmal metaphysisch.

Im ersten Fall würde das Verfahren zur Herstellung homöopathischer Arzneimittel in den illustren Kreis wissenschaftlicher Mythen aufgenommen. Gleichwohl gelte es, Hahnemann, den Begründer dieses Mythos, ins rechte Licht zu rükken. Denn auch wenn sein Suchen nach einer umfassenden Heilmethode ohne Erfolg geblieben ist, so entsprang diese Suche doch einer echten Not. Die Ärzteschaft seiner Zeit stand zu Recht in einem höchst zweifelhaften Ruf. Es ist eine Epoche der medizinischen Orientierungslosigkeit, die erst Mitte des 19. Jahrhunderts mit der endgültigen Ablösung der mehr als zweitausend Jahre vorherrschenden Humoralmedizin durch die Zellularpathologie Virchows eine Wende nimmt [14]. Auch heute hat die Medizin wieder eine Krise zu bestehen - wenn auch nicht wie damals in ihren Grundbegriffen selbst, so doch in ihrer vielleicht zu einseitig naturwissenschaftlich-technischen Ausrichtung (ohne hier aber die grossen Leistungen der modernen Medizin grundsätzlich in Frage stellen zu wollen), die allzuleicht vergessen macht, dass der Mensch in seinem Leben und Sterben mehr ist als ein in Einzelteile zerfallendes, verrechenbares Objekt.
Im zweiten Fall aber müsste man als Befürworter eines ganzheitlich-avantgardistischen Weltbildes wenigstens konsequent sein und an die Stelle des mechanischen Verfahrens der Arzneikraftentwicklung ein metaphysisches setzen. In diesen geistreichen Sphären, irgendwo zwischen Religion und Privatoffenbarung, wäre man endlich befreit von dieser unglückseligen irdischen Knechtschaft - der sinnlosen Suche nach wissenschaftlichen Beweisen.

\section{Literatur}

1 Hahnemann S. Organon der Heilkunst. Standardausgabe der 6. Auflage. Bearbeitet und herausgegeben von J. M. Schmidt. Heidelberg: Haug; 1996.

2 Lambeck M. Irrt die Physik? Über alternative Medizin und Esoterik. München: Beck; 2003.

3 Vithoulkas G. Die Wissenschaftliche Homöopathie. Theorie und Praxis naturgesetzlichen Heilens. 5. Auflage. Göttingen: Burgdorf; 1993.

4 Thurneysen A. Ist die Homöopathie eine energetische Therapieform? In: Heusser P (Hrsg.). «Energetische» Medizin. Gibt es nur physikalische Wirkprinzipien? Bern: Lang; 1998. S. 161-74.

5 Heusser P. Materielle und geistige Wirkprinzipien in der Anthroposophischen Medizin. In: Heusser P (Hrsg.). «Energetische» Medizin. Gibt es nur physikalische Wirkprinzipien? Bern: Lang; 1998. S. 191-205.

6 Kant I. Träume eines Geistersehers, erläutert durch Träume der Metaphysik. In: Kant I. Vorkritische Schriften bis 1768. Werkausgabe Band 2. 7. Auflage. Frankfurt am Main: Suhrkamp; 1991. S. 919-89.

7 Cassirer E. Gesammelte Werke. Bd. 12. Philosophie der symbolischen Formen. - Teil 2. Das mythische Denken. Hamburg: Meiner; 2002.

8 Kutschmann W. Die Newtonsche Kraft. Metamorphose eines wissenschaftlichen Begriffs. Wiesbaden: Steiner; 1983.

9 Holzhey H. Neukantianismus. In: Holzhey H, Röd W. Neukantianismus, Idealismus, Realismus, Phänomenologie. Geschichte der Philosophie. Hrsg. von Wolfgang Röd. Band XII. München: Beck; 2004. S. 11-129.

10 Cassirer E. Von Hegels Tod bis zur Gegenwart (1832-1932). Das Erkenntnisproblem in der Philosophie und Wissenschaft der neueren Zeit. Vierter Band. Hamburg: Meiner; 2000.

11 Kent JT. Prinzipien der Homöopathie. Schäftlarn: Barthel \& Barthel; 1996.

12 Beck A. Neuraltherapie als Informationsübermittlung. In: Heusser P (Hrsg.). «Energetische» Medizin. Gibt es nur physikalische Wirkprinzipien? Bern: Lang; 1998. S. 143-60.

13 Ausfeld-Hafter B. Das Konzept der Energie in der Traditionellen Chinesischen Medizin. In: Heusser P (Hrsg.). «Energetische» Medizin. Gibt es nur physikalische Wirkprinzipien? Bern: Lang; 1998. S. 175-89.

14 Schipperges H. Moderne Medizin im Spiegel der Geschichte. Stuttgart: Thieme; 1970. 